\title{
What Agreement Problems Owe Michel
}

\author{
Achour Mostefaoui \\ Ifsic/Inria, Université de Rennes, 35042 Rennes, France \\ achour@irisa.fr
}

Agreement problems are at the heart of the design of dependable and reliable distributed services. Distributed systems that run such services may experience unpredictable processing and communication delays, and some of their components can fail in various ways. It has been proved that in such settings, the consensus problem, the most popular and fundamental of the agreement problems has no deterministic solution.

Therefore, researchers started investigating ways of circumventing the impossibility result. Two main directions were explored: relaxing the requirements of the consensus problem, and strengthening the assumptions on the system. At least two ways of relaxing the consensus requirements have been investigated: randomization (termination is achieved only with high probability) and approximate agreement. Also, at least two ways of strengthening the assumptions on the system have been considered: adding synchrony assumptions to the system and abstracting the details of how a processor suspects a failure has occurred, without referring to particular synchrony assumptions by the mean of the Unreliable Failure Detectors that provides processes with a list of processes suspected to have crashed.

Michel Raynal has contributed to both directions and also to the combination of the two. He has co-authored more than a hundred papers on the topic. Moreover, he has with his co-authors initiated and investigated a new direction: the condition-based approach. The condition-based approach consists in looking at certain combinations of input values of a given distributed problem. It is often the case in practice that some combinations of the input values of processes occur more frequently than others.

After the specification of necessary and sufficient conditions that allow to solve some problems, a hierarchy of classes of conditions has been exhibited that allow to solve an agreement problem more and more efficiently when the condition is more constraining. Then, a first connection has been made with synchronous systems as the weakest condition that allows to solve consensus in the asynchronous model is the exact condition that allows to solve the same problem in a synchronous system but in the most efficient way revealing a continuum between synchronous and asynchronous systems. Moreover, a connection has been made between the Interactive Consistency agreement problem and errorcorrecting codes. Indeed, any error-correcting code can be used to parametrize a generic agreement protocol to solve the interactive consistency problem.

The talk will present the different facets of the contribution of Michel Raynal to the understanding of agreement problems (decidability, efficiency, algorithmic mechanisms, etc.). 